\title{
Preparation and optimization of nanoporous hollow carbon spheres /S composite cathode materials for Li-S battery
}

\author{
Wenhua Zhang ${ }^{1,3}$, Ping Liu ${ }^{2}$, Jiaxin Pan ${ }^{4}$, Xiaopin Yang ${ }^{1,3}$, Jia Liu ${ }^{1,3}$, Huijun X ${ }^{1,3}$, Zhizhao Ouyang ${ }^{1,3}$ \\ ${ }^{1}$ Nanchang Institute of Technology, Tianxiang Avenue No.289, 330099, Nanchang, China \\ ${ }^{2}$ State Grid Jiangxi Electric Power Company Limited research institute, Minqiang Road No.88, \\ 330096, Nanchang, China \\ ${ }^{3}$ Nanchang Institute of Technology, Jiangxi Province Key Laboratory of Precision Drive and Control, \\ Tianxiang Avenue No.289, 330099, Nanchang, China \\ ${ }^{4}$ Guangxi science and technology intelligence institute, Xinghu road No.24, 530022 Nanning, China \\ *E-mail: zhangwenhua_610@163.com
}

doi: $10.20964 / 2019.05 .52$

Received: 2 January 2019 / Accepted: 14 March 2019 / Published: 10 April 2019

\begin{abstract}
Aiming at the problems of dissolution and volume expansion of intermediates in lithium-sulfur batteries during the reaction process, a high-porosity mesoporous carbon sphere with hollow structure (labeled as PS-hollow carbon) is prepared by polystyrene(PS) template method. As a conductive matrix for sulfur electrode, it not only increases the sulfur load, but also adsorbs the polysulfide in the hollow structure of the sphere and the mesoporous pores of the shell. And with the $\beta$-Cyclodextrin as the sticking agent, the polysulfide ions are bound to the interior of hollow carbon by surface enveloping, which inhibits the dissolution of active materials. Scanning electron microscopy (SEM), transmission electron microscopy (TEM), and nitrogen adsorption and desorption characterization indicated that porous carbon has a hollow spherical structure with mesoporous pores(pore diameter of 2 4nm on the surface. The X-ray diffraction (XRD) pattern indicates that the elemental sulfur is uniformly dispersed in the hollow carbon pore structure. The thermogravimetric analysis showed that the sulfur content of PS-hollow carbon/S composite is $74.8 \mathrm{wt} . \%$. The electrochemical test showed that the as-prepared carbon/sulfur composite can deliver a reversible capacity of $1445 \mathrm{mAh} \mathrm{g}^{-1}$ at the first cycle, and remained above $750 \mathrm{mAh} \mathrm{g}^{-1}$ after 100 cycles, indicating that the PS-carbonized carbon/S composite has good electrochemical activity and cycle stability.
\end{abstract}

Keywords: High Pore Volume; Hollow Carbon Sphere; Carbon/Sulfur Composites; Li-S Battery 
(C) 2019 The Authors. Published by ESG (www.electrochemsci.org). This article is an open access article distributed under the terms and conditions of the Creative Commons Attribution license (http://creativecommons.org/licenses/by/4.0/). 